\title{
Pressure Induced Friction Collapse of Rare Gas Boundary Layers Sliding over Metal Surfaces
}

\author{
M. C. Righi* and M. Ferrario \\ CNISM-Consorzio Nazionale Interuniversitario per le Scienze Fisiche della Materia, \\ CNR-INFM National Research Center on nanoStructures and bioSystems at Surfaces $\left(S^{3}\right)$, Dipartimento di Fisica, \\ Università di Modena e Reggio Emilia, Via Campi 213/A, 41100 Modena, Italy
}

(Received 28 February 2007; published 24 October 2007)

\begin{abstract}
In this Letter we show that friction of anticorrugating systems can be dramatically decreased by applying an external load. The counterintuitive behavior that deviates from the macroscopic Amonton law is dictated by quantum mechanical effects that induce a transformation from anticorrugation to corrugation in the near-surface region. We describe the load-driven modifications occurring in the potential energy surface of different rare gas-metal adsorbate systems, namely, $\mathrm{Ar}, \mathrm{Kr}, \mathrm{Xe}$ on $\mathrm{Cu}(111)$, and $\mathrm{Xe}$ on $\mathrm{Ag}(111)$, and we calculate the consequent friction drop for the commensurate $\mathrm{Xe} / \mathrm{Cu}$ system by means of combined $a b$ initio and classical molecular dynamics simulations.
\end{abstract}

DOI: 10.1103/PhysRevLett.99.176101

PACS numbers: 68.35.Af, 61.50.Ks, 71.15.Mb

The atomic-scale control of sliding friction is a subject of growing importance. According to the time honored Amonton law, the friction force between two bodies is just proportional to the normal force-the applied load. However, that proportionality is normally related to the load dependence of the contact area [1]. At the microscopic level there is no guarantee for the Amonton law to hold in general and the system behavior is dictated by specific situations. While the rule is still that friction increases with load, it is possible to achieve microscopically some control over friction [2]. Here we find a direct, load-driven frictional drop for atomically thin boundary layers of rare gas monolayers pressed between flat metal surfaces. Monolayers (MLs) of rare gas atoms physisorbed on metallic surfaces have already received much attention both in surface science and in nanotribology. The frictional properties of $\mathrm{Ar}, \mathrm{Kr}$, and Xe MLs sliding inertially over the metallic surface of an oscillating quartz crystal microbalance $(\mathrm{QCM})$ have provided novel understanding of the fundamental laws of friction at the nanometer scale: these experiments constitute the first direct evidence of the existence of phononic friction [3]. Energy dissipation thorough phonons is dictated by the potential corrugation [4], i.e., how much the adsorption energy varies as an adatom moves from one site to another along the surface. The potential corrugation and its dependence on load is the key to our proposal and thus deserves a closer look.

The rare gas - metal surface interaction potential $V=$ $V(x, y, z)$ can be seen as the sum of two contributions [5]. At large atom-metal distances the potential $V$ is attractive, of van der Waals nature, proportional to $\left(z^{-3}\right)$ [6]. At short range, the individual metal atoms and their charge distributions overlap with the rare gas closed shell turning the interaction from attractive to repulsive. If the adatomsurface interaction is generated by empirically summing pair potentials, then the hollow adsorption sites prevail over top sites. However, several experimental studies brought out that in reality the top sites are minima for rare gas adsorbates on metal surfaces [7]. The origin of this so-called anticorrugation effect has been widely debated, recently a novel interpretation has been proposed on the basis of first-principles calculations [8]. In this Letter we will focus on the load dependence of anticorrugation and its direct impact on the tribological properties of sliding MLs. We will show that the anticorrugation diminishes, instead of increasing under load, crossing zero, and reverting to corrugation above a critical pressure, $P_{c}$. Our main point will be that the sliding friction coefficients of an anticorrugating system should drop with pressure, hitting a minimum in correspondence to $P_{c}$, and rising again after that.

In order to realistically describe the rare gas-metal surface interaction we performed ab initio calculations for $\mathrm{Ar}$, $\mathrm{Kr}, \mathrm{Xe}$ adsorption on $\mathrm{Cu}(111)$ and $\mathrm{Xe}$ on $\mathrm{Ag}(111)$ in the $(\sqrt{3} \times \sqrt{3}) R 30^{\circ}$ ( $\sqrt{3}$ from now on) structure. An analytical expression which describes the potential energy surface (PES) experienced by the rare gas atom on the metal was derived, taking as reference the $\mathrm{Xe} / \mathrm{Cu}$ system, according to the following procedure. We sampled the adatom physisorption well at different lateral positions $(\bar{x}, \bar{y})$ on the surface by calculating the adatom adsorption energy $V(\bar{x}, \bar{y}, \infty)-V(\bar{x}, \bar{y}, z)$ at different vertical distances $z$. The results were fitted around the minimum in the form: $f(z)=A_{0} \exp \left(-z / A_{1}\right)-A_{2} / z^{3}$, the origin of which has been explained earlier, the repulsive part mimicking the exponential decay of the surface charge density into the vacuum. As can be seen from Fig. 1, the fitting is very satisfactory in the near-surface region, while it departs from the density-functional theory (DFT)-local-density approximation (LDA) points at long range, due to the presence of the $z^{-3}$ term, which improves the description of the long-range interaction with respect to the DFT-LDA. In this manner we have obtained for every $(\bar{x}, \bar{y})$ grid point a set of parameters $\left(A_{0}, A_{1}, A_{2}\right)$ characterizing the adatom physisorption well at that site. As the $A_{i}$ parameters are $2 \mathrm{D}$ periodic functions, they have been expanded in the Bloch 


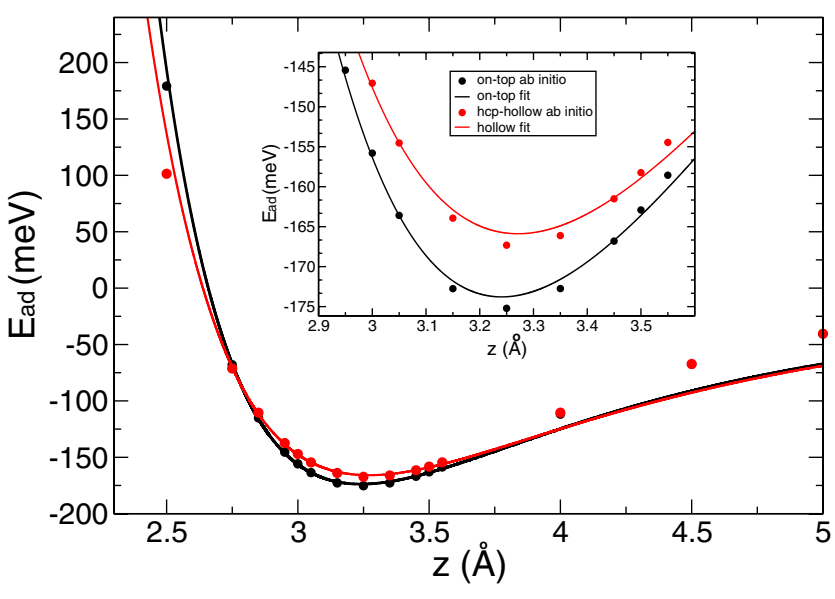

FIG. 1 (color online). Adsorption energy for one Xe adatom deposited on the $\mathrm{Cu}(111)$ surface as a function of its distance $(z)$ from the outermost $\mathrm{Cu}$ layer. The ab initio results (dots) and the parametric functions described in the text (full lines) are reported both for Xe adsorption at the on-top and at the hcp-hollow sites.

form $A_{i}(x, y)=A_{i}^{\text {top }}+\Delta_{i} u(x, y)$, where $u(x, y)$ is a periodic function with the symmetry of the surface, $\Delta_{i}=$ $A_{i}^{\text {ho }}-A_{i}^{\text {top }}$ measures the amplitude for the $i$ th parameter swing between the top site value and hollow site value. In this way we obtained a three-dimensional potential

$$
V(x, y, z)=A_{0}(x, y) \exp \left[-z / A_{1}(x, y)\right]-A_{2}(x, y) / z^{3},
$$

which describes the interaction of a rare gas atom and a metal surface. As anticipated, it is anticorrugated with minima at top sites, saddle points at bridge sites, and maxima at hollow sites [9]. A three-dimensional representation of the PES derived for the $\mathrm{Xe} / \mathrm{Cu}$ adsorbate system is given in Fig. 2. Panel (a) shows the energy landscape seen by a $\mathrm{Xe}$ atom at the equilibrium distance from the $\mathrm{Cu}(111)$ surface. By decreasing the adatom-surface separation below this value, emulating what happens under an applied load, the anticorrugation gradually decreases until the potential energy surface becomes essentially flat (panel b). A further decrease of the adatom-surface separation causes corrugation to reappear (panel c) no longer "inverted"; the hollow sites are now absolute minima and the top sites maxima. In this regime the delicate physics underlying anticorrugation, addressed and discussed in great detail by several authors, is as if it was "squeezed out" by pressure.

The critical distance $z_{c}$ where the PES flattens corresponds to the adatom-surface separation at which the curves of Fig. 1, representing the on-top and the hollow physisorption wells, cross. Such potential crossing has been noted also for $\mathrm{Xe}$ on $\operatorname{Pt}(111), \operatorname{Pd}(111)$ [8,12], and for $\mathrm{He}$ on $\mathrm{Cu}(111)$ [13]. Our results for $\mathrm{Ar}, \mathrm{Kr}$ on $\mathrm{Cu}(111)$, and for $\mathrm{Xe}$ on $\mathrm{Ag}(111)$ suggest that this is a general feature for rare gas adsorption on metals. The on-top and the hollow physisorption wells, calculated for these systems by fitting the $a b$ initio energetics at the two symmetry sites, present, in fact, the same crossing seen in Fig. 1 for the $\mathrm{Xe} / \mathrm{Cu}$ case. What characterizes each system is the critical adatom-surface distance $z_{c}$ at which such crossing occurs: we found $z_{c}=2.47 \AA$ for $\mathrm{Ar} / \mathrm{Cu} ; z_{c}=2.63 \AA$ for $\mathrm{Kr} / \mathrm{Cu}$; $z_{c}=2.78 \AA$ for $\mathrm{Xe} / \mathrm{Cu}$; and $z_{c}=3.12 \AA$ for $\mathrm{Xe} / \mathrm{Ag}$. A quick look at tables of atomic radii reveals that $z_{c}$ closely resembles the sum of the atomic radius of the adatom and the radius of the atomic core of the metallic element constituting the substrate. This suggests that the hollow site becomes favored over the on-top site when the rare gas and metal closed shells start to overlap: in this regime, geometrical reasons causes Pauli repulsion to be lower at the interstitial hollow site than at the on-top one, as a hardsphere model of the system would suggest.

The next point we will address regards the implications that anticorrugation and its pressure-induced transformation to corrugation have on the tribological properties of rare gas on metals. We will focus our attention on the $\mathrm{Xe} / \mathrm{Cu}$ adsorbate system, which, on the contrary of the other considered cases, is a commensurate system where friction is higher and the pressure effects described in the following are more important. At $77 \mathrm{~K}$, Xe forms a commensurate $\sqrt{3}$ structure on $\mathrm{Cu}(111)$ [14]. This perfect commensurability implies a very strong pinning, and a large static friction for a defect free layer. The restoring force acting on a particle that moves along the potential profile in the [110] direction, the $x$ direction, is represented for decreasing values of $z$ in Fig. 3 (lines). It was obtained by calculating the values assumed by the $x$-partial derivative of Eq. (1) at $(x=a / 4, y=0, z)$, i.e., where the potential profile is steepest. At $z_{\text {eq }}$ it is about $9 \mathrm{meV} / \mathrm{A}$, or $14 \mathrm{pN}$, per Xe adatom. By decreasing $z$, the potential profile smoothes out and the static friction force drops, hitting a nominal value of zero at $z_{c}$. For quotas $z$ lower than $z_{c}$, the PES becomes corrugated with particles occupying the new minima at the hollow sites. The dashed line of Fig. 3 represents the restoring force for a particle moving along a straight path in the $x$ direction connecting two adjacent hollow sites [see Fig. 2(c)]. In order to consider a realistic situation, we carried out molecular dynamics simulations designed to calculate the static friction at $77 \mathrm{~K}$, which is the temperature often considered in QCM experiments. We considered a ML of 2048 Xe atoms interacting among them via a Lennard-Jones potential $(\sigma=3.95 \AA, \epsilon=20 \mathrm{meV})$. The interaction with the $\mathrm{Cu}(111)$ surface was modeled by the ab initio derived potential of Eq. (1). Equilibrium temperature was controlled by means of a Nosé-Hoover thermostat. After initial annealing at zero force, here the Xe atoms settled, defect free, in full registry with the $\sqrt{3} a_{\mathrm{Cu}}$ surface lattice, a lateral force $F_{x}$ was adiabatically applied to all the particles. The force was increased until the Xe monolayer center of mass velocity became nonzero, signaling depinning. The simulation was then repeated for different applied vertical loads $F_{z}$. The results (circles in Fig. 3) confirm the pressureinduced drop of static friction, until about $P_{c}=5 \mathrm{GPa}$. For 

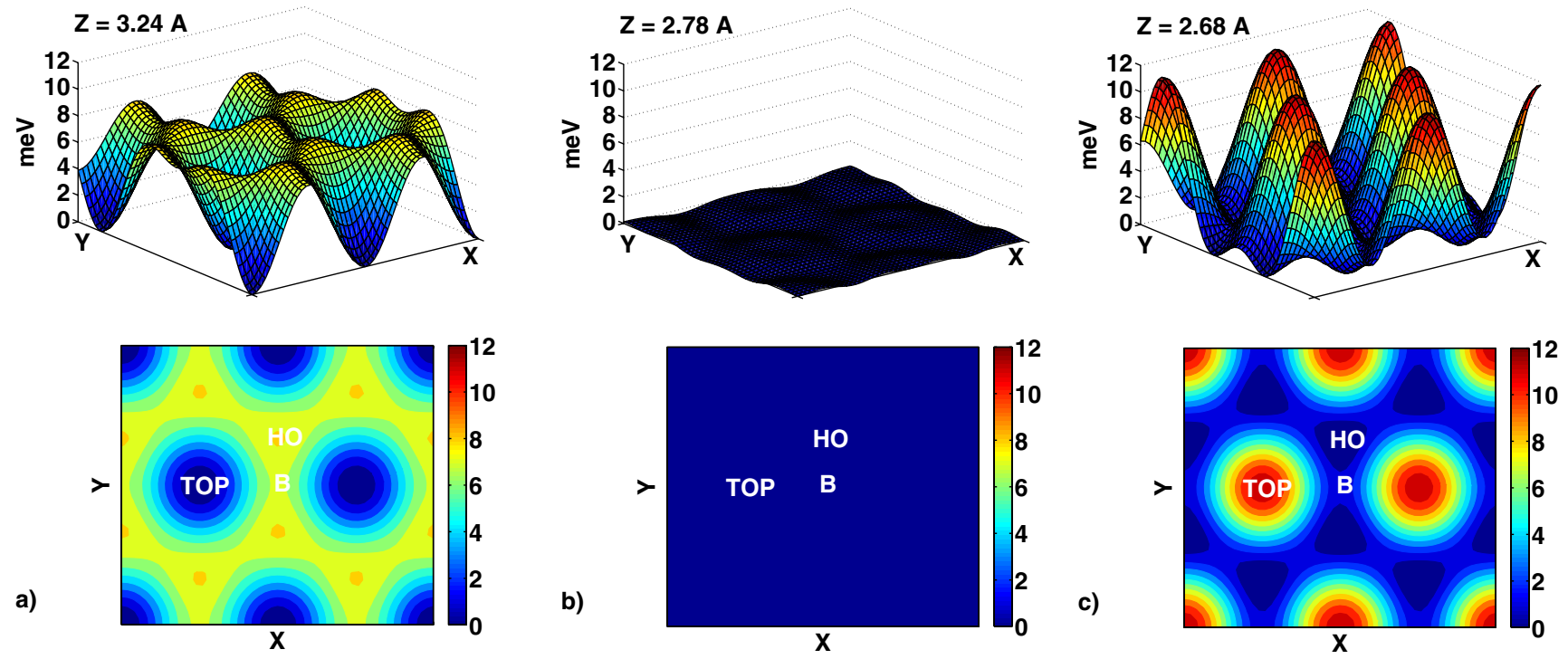

FIG. 2 (color online). The $V(x, y, z)$ function of Eq. (1), which represents the potential energy landscape experienced by a Xe atom on $\mathrm{Cu}(111)$, is displayed for different values of the adatom-surface distance $z$. By decreasing $z$ from its equilibrium value (a), the PES smooths out until it becomes almost flat (b), and, subsequently, an inverse corrugation appears (c). It is worth noticing that not only the PES corrugation, but the PES shape by itself undergoes a modification in passing from the anticorrugated (a) to the corrugated (c) regime.

higher pressures, the static friction forces obtained by the molecular simulation deviates significantly from the restoring forces calculated analytically along the $x$ direction. A closer analysis revealed that the particle jumps which give rise to the locked-to-running transition of the ML occur along the path whose endpoints are hollow sites, but which passes through a PES saddle point (a bridge site). As can be seen in the bottom part of Fig. 2(c), this path forms a $30^{\circ}$ angle with the $x$ direction. A similar particle behavior has been advocated in Ref. [15] to explain the two-dimensional nature of the atomic-scale friction measured by means of a friction-force microscope. This result revealed that even in the presence of an external force, applied along a specific direction, particle movements inside the ML follow the minimum energy path, as it happens in ordinary surface diffusion. Thus, the key quantity to be monitored in order to get information relevant for the ML friction, is the height of the PES saddle points and not the height of the PES maxima relative to the PES minima as it has often been considered. In our specific case, the symmetry of the triangular lattice causes the PES saddle point to correspond to the bridge sites both in the anticorrugated and the corrugated regime, with a significant difference in energy which is close to that of the PES maxima in the first case, and that of the PES minima in the second case (see Fig. 2). This difference gives rise to a different particle dynamics in the two situations.

In order to investigate the effect of pressure on friction in a kinetic situation, we considered a rare gas ML sandwiched between two metallic surfaces in relative motion, each of them represented by the washboard potential of Eq. (1). The interaction between the two metals was not taken into account at this stage, thus the considered system could be representative of a situation of boundary lubrication if the rare gas lubricant indeed isolates the two metallic surfaces. The top surface was allowed to move along the

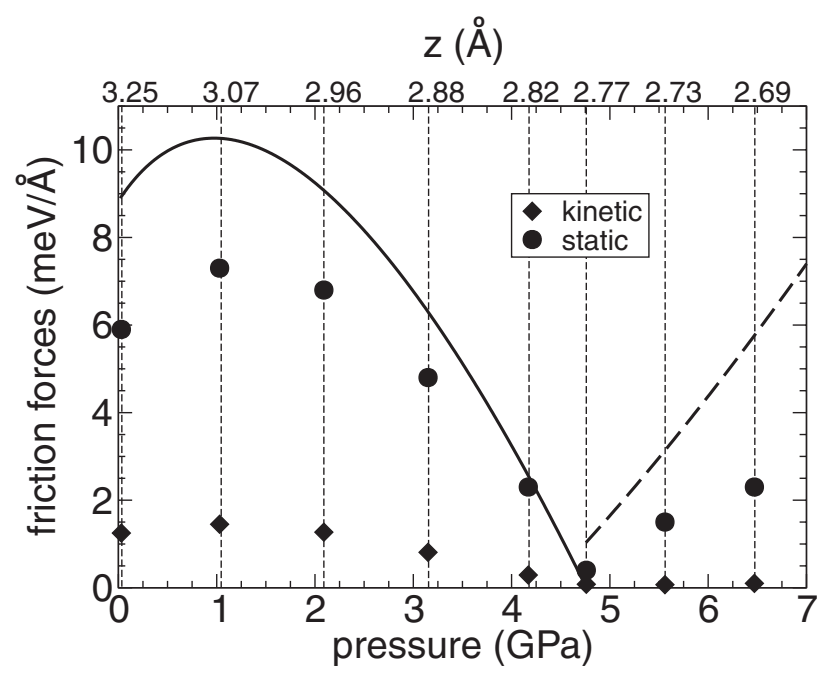

FIG. 3. Pressure-induced friction drop calculated for a commensurate $\mathrm{Xe} \mathrm{ML}$ on the $\mathrm{Cu}(111)$ surface. The lines represent the restoring force (see text) acting on a particle that moves straight along the [110], the $x$ direction, calculated as a function of pressure. The full line refers to an anticorrugated PES [Fig. 2(a)], the dashed line to a corrugated one [Fig. 2(c)]. The symbols represent the static (circles) and the kinetic (diamonds) friction force obtained by classical molecular dynamics simulations at $T=77 \mathrm{~K}$. The ML-surface distance corresponding to the different pressures we considered is also reported. 
$x$ direction with a constant velocity $v$ relative to the substrate which was considered at rest. The simulation, performed at a constant temperature of $77 \mathrm{~K}$, covered a time interval of $5 \mathrm{~ns}$. In order to achieve meaningful averages one needs that the top surface spans a reasonable number of lattice periods during this time interval. We chose $v=$ $10 \mathrm{~nm} / \mathrm{ns}$, a velocity which is large compared to the sliding velocities usually detected in QCM experiments (of the order of few $\mathrm{cm} / \mathrm{s}$ [1]), but still much lower than thermal velocities; thus we expect our results (obtained as time averages) to be representative of system dynamics under the effect of a lower external velocity on a longer time scale. The simulation was repeated for different space separations between the two metal surfaces to investigate the effect of the applied pressure. In all the cases we considered, we found for the ML center of mass position that $z_{\mathrm{cm}}$ corresponds to half of the spacing between the metallic surfaces, $y_{\mathrm{cm}}$ oscillates around its initial value, and $x_{\mathrm{cm}}$ increases linearly with time. The slope of $x_{\mathrm{cm}}(t)$ indicates a ML drift velocity equal to $v / 2$. During the simulation, the forces acting on the particles due to the interaction with the sliding top surface and with the fixed substrate were recorded. By time averaging the recorded values, we found that $\left(F_{\alpha}^{\mathrm{top}}=-F_{\alpha}^{\mathrm{sub}}\right)$ for all three components $\alpha=x, y, z$. In particular, $F_{y}=0$ (with some small deviations in the corrugated regime), while $F_{x}$ varies as a function of the applied pressure as indicated by the diamondlike symbols of Fig. 3 (the pressure corresponding to each ML-surface distance-reported in the upper part of Fig. 3-was obtained by dividing the value of $F_{z}$ observed at that distance by the area per particle in the ML). We can see that $F_{x}$ decreases as a function of pressure; thus, the ML kinetic friction undergoes the same pressure-induced reduction observed for its static friction. $F_{x}$ can also be interpreted as the force that it is necessary to apply in order to have the top metal surface moving on average at constant velocity, i.e., the friction force acting on a metallic surface lubricated by rare gases. The observed load-driven reduction of friction may suggest the use of "anticorrugating" species as lubricant in conditions of boundary lubrication where pressures are in the range of GPa. This aspect should, however, been further investigated by means of simulations which take into account the deformation of the metallic surfaces.

In this Letter we discussed the possibility of controlling the shape of the potential energy surface of anticorrugating systems by applying an external load and its consequences on the frictional properties of these systems. By means of classical molecular dynamics simulations, relying on $a b$ initio derived potentials, we calculated a pressureinduced drop of the static and the kinetic friction of the $\mathrm{Xe} / \mathrm{Cu}(111)$ commensurate system. According to Amonton law, friction forces should increase linearly with load. Our results reveal that this general behavior does not hold for anticorrugating systems on a scale where the mechanism of increase of contact area cannot be advo- cated and quantum mechanical effects prevail. For the chosen example, the uniaxial stress required (few GPa) for friction disappearance probably hinders practical applications; however, many other systems present on-top adsorption at equilibrium, for which hollow adsorption could become favored under pressure at more suitable conditions for practical application. Finally, our analysis on particle dynamics in both the anticorrugated and in the corrugated regimes revealed that at an atomic scale the friction force can assume components along directions different from the sliding direction, as assumed for the friction force at a macroscopic level. This is due to the fact that particle movements inside the ML follow the minimum energy path. Thus, the relevant barriers for friction dissipation are those defined by the PES saddle points.

We are grateful to Virginio Bortolani, Luigi Maurichi, Erio Tosatti and Andrea Vanossi for stimulating collaboration on this subject. This research was supported by MIUR Cofin No. 2004023199.

*mcrighi@unimore.it

[1] B. N. J. Persson, Sliding Friction: Physical Principles and Applications (Springer, New York, 2000), 2nd ed.

[2] A. Socoliuc, E. Gnecco, S. Maier, O. Pfeiffer, A. Baratoff, R. Bennewitz, and E. Meyer, Science 313, 207 (2006).

[3] J. Krim, D. H. Solina, and R. Chiarello, Phys. Rev. Lett. 66, 181 (1991).

[4] M. Cieplak, E. D. Smith, and M. O. Robbins, Science 265, 1209 (1994).

[5] L.W. Bruch, M.W. Cole, and E. Zaremba, Physical Adsorption: Forces and Phenomena (Clarendon, Oxford, 1997).

[6] E. Zaremba and W. Kohn, Phys. Rev. B 13, 2270 (1976).

[7] R. D. Diehl et al., J. Phys. Condens. Matter 16, S2839 (2004).

[8] J. L. F. Da Silva, C. Stampfl, and M. Scheffler, Phys. Rev. Lett. 90, 066104 (2003); Phys. Rev. B 72, 075424 (2005).

[9] Further details on the $a b$ initio calculations and on the parametrization of the rare gas-metal surface interaction potential can be found in Ref. [10]. We anticipate here that the use of LDA, which overestimates bonding, can produce a consequent overestimation of the potential corrugation [11]. It is important to appreciate, however, that the accuracy of LDA increases rapidly with decreasing interatomic distances, and the effects described in this Letter are dominated by the short-range interactions.

[10] M. C. Righi and M. Ferrario, J. Phys. Condens. Matter 19, 305008 (2007).

[11] P. Lazic, Z. Crljen, R. Brako, and B. Gumhalter, Phys. Rev. B 72, 245407 (2005).

[12] J. A. Barker, C. T. Rettner, and D. S. Bethune, Chem. Phys. Lett. 188, 471 (1992).

[13] N. Jean, M. I. Trioni, G. P. Brivio, and V. Bortolani, Phys. Rev. Lett. 92, 013201 (2004).

[14] J. Jupille, J. J. Ehrhardt, D. Fargues, and A. Cassuto, Faraday Discuss. Chem. Soc. 89, 323 (1990).

[15] S. Fujisawa, E. Kishi, Y. Sugawara, and S. Morita, Phys. Rev. B 51, 7849 (1995). 